\title{
LOGIT MODEL ANALYSIS FOR PAIRWISE COMPARISONS IN RATIO SCALE
}

\author{
Indrani Basak and Prasanta Basak \\ Department of Mathematics, Penn State Altoona \\ 120 Eiche \\ 3000 Ivyside Park \\ Altoona PA 16601-3760 \\ fkv@psu.edu
}

\begin{abstract}
Pairwise comparison methods involving ratio scale models are considered. The pairwise comparison values sometimes are cardinal representations of ordered categorical variables. This model arises in Saaty's scaling method of priorities which is referred to as Analytic Hierarchy Process (AHP). In this article, we develop a logit model for the ordered response variable in the AHP. Relevant estimation and hypothesis testing procedures are addressed in this perspective.
\end{abstract}

